\title{
Carnets
}

Revue électronique d'études françaises de l'APEF

Deuxième série - 2 | 2014

Frontières de la chronique

\section{Écrire le passé en compilant le présent}

Le Mercure François, atelier d'écriture de l'histoire du temps présent

Virginie Cerdeira

\section{OpenEdition}

Journals

Édition électronique

URL : http://journals.openedition.org/carnets/1266

DOI : $10.4000 /$ carnets. 1266

ISSN : 1646-7698

Éditeur

APEF

Référence électronique

Virginie Cerdeira, «Écrire le passé en compilant le présent », Carnets [En ligne], Deuxième série -

2 | 2014, mis en ligne le 30 novembre 2014, consulté le 05 mai 2019. URL : http://

journals.openedition.org/carnets/1266; DOI : 10.4000/carnets.1266

Ce document a été généré automatiquement le 5 mai 2019.

\section{(c) (i) (8)}

Carnets est mis à disposition selon les termes de la licence Creative Commons - Atribution - Pas d'utilisation commerciale 4.0 International. 


\title{
Écrire le passé en compilant le présent
}

Le Mercure François, atelier d'écriture de l'histoire du temps présent

\author{
Virginie Cerdeira
}

\section{NOTE DE L'AUTEUR}

Cet article est tiré du travail réalisé dans le cadre d'une thèse d'histoire moderne en cours sur Le Mercure François et les usages politiques de l'écriture du passé à l'Université d'AixMarseille sous la direction de Guy Le Thiec et d'Isabelle Luciani commencée en septembre 2011.

\section{Introduction}

1 Le 29 novembre 1610, le pouvoir royal délivre à Jean Richer, imprimeur-marchandlibraire en l'Université de Paris le monopole commercial de la diffusion du Mercure François, ou la suitte de l'Histoire de la paix [...] (MF vol. I, 1611). En renvoyant à l'ouvrage de Palma-Cayet, historien-chronologue depuis 1596 (Yardeni, 1981:292), le rédacteur du Mercure inscrit son ouvrage dans la tradition de l'écriture historiographique et pose la question de ses rapports avec la chronique et autres annales ${ }^{1}$. La plasticité des formes de l'écriture historiographique, aux normes et règles sans cesse redéfinies et réaffirmées par les praticiens et théoriciens de l'écriture du passé pose la question de la situation du Mercure François dans cette nébuleuse générique (Guenée, 2011:207). La mise en place d'une périodicité de la publication dès la deuxième livraison en 1613 ainsi que la constitution d'une collection de 25 volumes publiés entre 1611 et 1648 accentuent cette interrogation en rapprochant le Mercure François de la presse. La mise en texte et en imprimé du Mercure implique la rencontre et l'exploitation de temporalités multiples. Dans la lignée de la tradition historiographique, le rédacteur fait son miel des actualités mises sous presse dans des libelles, occasionnels ou édits royaux en les compilant afin 
qu'elles forment une histoire. Ce faisant, le Mercure éloigne le passé proche pour en faire un passé antérieur et fabrique presque immédiatement une mémoire historique et politique au royaume.

2 Alors que le Mercure François occupe cette position nodale au cœur des temporalités, quelle est la part prise par les emprunts aux genres historiques, en particulier la chronique, dans l'écriture d'une histoire du temps présent ? La situation particulière du Mercure pose la question de l'héritage de la tradition de la chronique ainsi que de l'élaboration d'une mise en texte et en imprimé spécifiques. Le Mercure est-il susceptible d'avoir fondé un genre à lui seul quitte à en être l'unique représentant? Faut-il alors soutenir la tendance nominaliste jusqu'à considérer que la notion de genre est dépourvue de pertinence heuristique ${ }^{2}$ ? Les regards - internes ou externes - portés sur le Mercure François proposent d'esquisser les limites du genre et d'y repérer la place prise par le passé, en lien avec sa mise en ordre par l'écriture. La définition du Mercure portée par son propre péritexte se trouve confrontée aux pratiques d'écriture effectivement mises en œuvre dans le périodique ainsi qu'aux critiques des contemporains. Ces derniers opposent au Mercure des normes attendues en vertu de l'appartenance au genre historique, affichée par le titre de l'ouvrage. C'est que, nous rappelle Hans-Robert Jauss, le genre suscite des attentes auprès des lecteurs (Jauss, 1988 : 365-366). Les critiques des contemporains ou la malléabilité de l'œuvre s'expliquent-elles parce que le Mercure appartiendrait à un genre mal identifié ? Celui de l'histoire du temps présent ? Ou encore celui des Mercures? Il semble que le destin du Mercure François le range dans la catégorie des œuvres, si ce n'est des genres caducs (Montandon et Neiva, 2014 : 9-16). L'évolution de la mise en écriture du passé au gré des livraisons comme du contexte politique questionne les usages politiques de la chronique et du passé. À côté de la conception naturaliste de l'évolution des genres élaborée par Ferdinand Brunetière (Schaeffer, 1989 : 51-52), est-il possible d'expliquer celle du Mercure François par les circonstances politiques qui sont celles de sa rédaction et de sa publication? Autrement dit, n'est-il pas souhaitable d'examiner les transformations de la publication à l'aune « (...) des situations historiques et concrètes (...) qui ont pu (...) conditionner ce processus » (Jauss, 1986 : 52) et donc de contextualiser l'objet littéraire et ses formes ${ }^{3}$.

\section{Regards sur le Mercure François. Éléments de définition générique}

\section{Déclaration(s) d'intention(s) : généalogie et péritexte}

Lorsqu'il publie le premier volume du Mercure François en 1611, Jean Richer s'adresse à ses lecteurs dans une " Preface au lecteur » (MF vol. I, 1611 : sans foliotation, s.f.). Il y propose des éléments de définition du genre du Mercure. La tâche est nécessaire puisque les praticiens de l'écriture du passé eux-mêmes rencontrent de nombreuses difficultés à établir clairement ce qui différencie les genres historiques tels que les histoires, annales, éphémérides ou autres chroniques. C'est le cas depuis l'Antiquité (Guenée, 1973 : 1000). Les distinctions se sont affinées au fil des siècles et les ajustements concernent la place prise par la chronologie, le récit historique, le statut du témoin ou encore les commentaires c'est-à-dire l'interprétation des faits. Théoriciens et praticiens de l'écriture du passé se contredisent et entrent en désaccord, rendant nécessaire les redéfinitions récurrentes des limites entre chaque genre. Celles-ci ne sont d'ailleurs pas toujours 
posées et les débuts de l'époque moderne voient un usage indifférencié des termes de chroniques, annales ou histoires qui deviennent presque des synonymes. L'analyse du péritexte du Mercure François permet de mesurer les conceptions de son rédacteur à cet égard. Avant de revenir sur le texte de la préface, il convient de noter que le titre complet de la première livraison du Mercure affirme que c'est un livre d'histoire. Cette histoire est celle de la paix sous le règne d'Henri IV et jusqu'au sacre de son fils Louis XIII. Elle se veut la suite de la Chronologie septenaire de l'Histoire de la paix entre les Roys de France et d'Espagne (Cayet, 1605). Le titre choisi par Jean Richer indique qu'il se réclame de l'œuvre de PalmaCayet. Ce dernier précise le pacte de lecture qu'il propose à ses lecteurs au moyen du titre de son ouvrage comme de l'épître au roi (Cayet, 1605 : s.f.). La double épître (au roi puis au gouverneur de Bourgogne) de Cayet lui permet de revenir sur le flou entretenu par son titre. Son livre se veut, en effet, la Chronologie septenaire de l'Histoire de la paix entre les Roys de France et d'Espagne. Le double recours aux termes d'histoire et de chronologie lui évite d'avoir à déterminer fermement le genre de son livre. La notion de chronologie - qui ne recouvre pas exactement celle de chronique - renvoie à l'exigence d'ordonner le temps selon l'ordre chronologique, et ce de manière parfois tout à fait sommaire puisqu'il peut s'agir de simples listes ou tableaux chronologiques (Guenée, 1973: 1006). La notion d'histoire implique, quant à elle, un récit développé des faits allant au-delà de leur simple recensement. Elle pourrait supposer la présence, au moins occasionnelle, de commentaires (Guenée, 2011, 37-38). Peut-être en raison de ce flou, Cayet tient à revenir sur les déclinaisons du genre historique.

Ce terme de Chronologie est different de l'Histoire, des Annales, des Chroniques, \& de l'Ephemeride: car en toutes ces façons de tiltres sont les escrits des choses memorables advenuës de tous temps, en tous peuples \& nations, \& par toutes manieres d'Autheurs, selon que chacun a pris plaisir plutost en une sorte qu'en l'autre \& selon le subjet des matieres. (Cayet, 1605 : s.f.)

Cayet de poursuivre le panorama des genres historiques afin de justifier et valoriser le choix de la chronologie, qui sous sa plume et sous les presses de Richer fait figure de " genre historique total $»^{4}$ :

(...) l'Histoire est un recit des choses que l'Autheur a veues, \& les sçait pour les avoir veües (...). Les Annales au contraire sont un bref recit de quelques accidents particuliers (...). Les Chroniques recherchent le temps immemorial, \& dez la premiere antiquité \& fondation des peuples. (...) L'Ephemeride en fin est ce qui s'est faict \& dict de personne à personne (...). Mais la Chronologie porte en elle toutes les considerations des susdictes sortes \& manieres d'escrire les choses qui se passent, combien qu'en toutes occurrences il n'est pas besoin ny necessaire que le Chronologue ait esté present par tout (...). (Cayet, 1605 : s.f.)

5 L'ambiguïté demeure puisque, quelques lignes plus loin, Cayet affirme que c'est la méthode de la chronologie qu'il a adoptée pour cette « (...) Histoire presente de la paix (...) ». Qu'est-ce à dire ? L'ouvrage de Cayet présente-t-il en réalité une histoire dont la matière ne saurait uniquement résider sur le témoignage oculaire de son auteur? En continuant à associer les termes de chronologie et d'histoire après les avoir distingués, Cayet définit son ouvrage sans le définir.

Que conserve Jean Richer de ces considérations dans son propre péritexte? Il ne reprend pas le propos de Cayet, sans doute parce qu'il y adhère et s'inscrit dans sa ligne éditoriale. En revanche, il trace une frontière générique entre son texte et les panégyriques. Il exclut dès lors son Histoire de la paix des genres littéraires, notamment des genres rhétoriques recensés par Aristote (Maingueneau, 2007 : 29). Les dissemblances portent sur le style et 
le registre. L'histoire suppose une éthique de la vérité ; qui implique la simplicité du propos:

Je ne te donne point un Panegyre eloquent au lieu d'une Histoire, ny de grands discours philosophiques enrichis aux bordages de tout ce que les autheurs Grecs et Latins ont escrit de plus beau; ains seulement une simple narration de ce qui est advenu aux six annees dernieres (...) (MF vol. I, 1611 : s.f.).

7 L'histoire, qui s'écrit avec la double exigence de l'Alithie (la vérité) et de l'Apathie (la neutralité) trouve dans le propos simple la garantie à ces principes éthiques (Cayet, 1605 : s.f. et MF vol. I, 1611 : s.f.). En cela, elle se rapproche de la chronique qui comme l'histoire propose pendant longtemps un récit à la narration dépourvue d'ornements rhétoriques voire un simple tableau chronologique (Guenée, 2011: 203). Pendant longtemps ${ }^{5}$, la différence entre histoire et chroniques est de degré et non de nature (Guenée, 1973: 1015). L'histoire n'est pas plus éloquente que la chronique - pour nos deux auteurs - mais elle est plus développée (chaque livraison du Mercure compte environ un millier de pages). En relatant des faits survenus dans un passé proche par le recueil et la compilation d'actualités, le Mercure François entre dans le domaine du politique. C'est une mémoire historique des années de paix du royaume depuis 1598 qu'écrivent les deux ouvrages successifs (celui de Cayet puis de Richer) ${ }^{6}$. Le pouvoir politique ne peut être indifférent à la construction mémorielle proposée par les spécialistes de l'écriture du passé et à ses effets potentiels sur le présent et le futur du royaume.

\section{Quelle langue pour écrire le passé ?}

8 Pour le Sénat de Cologne qui revient sur la première livraison du périodique, le Mercure François appartient à la catégorie des "annales" (BnF, Richelieu manuscrits, fonds français, Cinq-Cents Colbert). Peut-être parce que perdure pour l'institution une définition médiévale des différents genres historiques selon laquelle: "L'orateur ou historien se distinguait du chroniqueur ou annaliste en ceci que le premier maniait le latin avec élégance, tandis que le second soit impuissance soit modestie, se résignait à dire les faits dans une langue vernaculaire sans apprêts. » (Guenée, 1973 : 1012). Le choix de la langue vernaculaire, commun à la Chronologie septenaire et au Mercure François, suppose des opérations de traductions du latin vers le français (MF vol. 1, 1611: s.f.) et rapproche les deux ouvrages de la chronique et des annales; alors que le recours à un récit développé les en éloigne (Guenée, 1973 : 1001). Peut-être est-ce la raison de la double détermination proposée par Cayet dans son titre ou de la présence du qualificatif « françois » dans le titre du périodique. La posture linguistique commune aux deux textes a des retombées politiques en matière de diffusion et de réception. En optant pour la référence au messager des dieux, le Mercure François ne se réclame pas uniquement de la Chronologie de Cayet mais aussi du Mercurius Gallobelgicus ${ }^{7}$. Il propose un travail de collecte et de rediffusion identique à celui pratiqué dans le périodique impérial en y ajoutant une dimension de vulgarisation nationale par le choix de la langue. Le legs linguistique de la chronique et des annales trouve son explication dans la volonté politique de participer à l'élaboration d'une mémoire du royaume. Ce choix peut aussi s'expliquer, toujours d'un point de vue politique, dans l'éveil d'une conscience nationale à l'issue de la période des guerres de religion dont l'une des modalités est la préférence pour la langue vulgaire (Yardeni, 1971).

9 C'est pour cela que le pouvoir royal surveille les éléments mémoriels qui trouveront leur place dans une histoire écrite, livraison après livraison, pour la postérité. 


\section{La périodicité de la publication : invention ou tradition?} qu'est inventée la périodicité de l'ouvrage. Cette découverte est prétendue fortuite, comme si en publiant la suite de l'ouvrage de Cayet, Jean Richer puis son frère Estienne s'étaient rendus compte qu'il était possible de faire perdurer l'entreprise ${ }^{8}$. C'est ainsi que débute l'adresse du « Libraire au Lecteur » du deuxième volume du Mercure :

CESTE Continuation du Mercure François m'estant tumbee entre les mains, J'ay pensé qu'en l'imprimant tu la recevrois d'aussi bon œil que le Mercure (...) (MF vol. II, 1613 : s.f.).

11 Le flux constant du temps, l'activité incessante qu'il génère et les usages politiques de sa mise en ordre par l'écriture expliquent la découverte de la périodicité. Cette invention est antérieure au Mercure François. Si l'histoire implique qu'elle soit écrite par un contemporain (Cayet, 1605 : s.f.), l'historien a toujours du matériau à traiter : «Limité au passé, l'historien entend du moins le couvrir tout entier. Dans ce tissu continu qu'est le temps passé, le seul moment décisif est la mort de l'historien. Tant qu'il vit, aucune raison de ne pas poursuivre son récit." (Guenée, 2011:22). En instaurant une publication périodique, le Mercure pérennise à la fois la narration entamée avant 1610 et la tradition médiévale qui fait de l'historien l'écrivain d'un continuum temporel. Il est donc sans cesse à l'œuvre et ses publications se succèdent. Le Mercure François ne déroge pas à la règle en proposant une Continuation du Mercure François ou suitte de l'Auguste Régence (...) (MF vol. II, 1613). La prolongation du récit continu au-delà de la vie d'un seul historien n'est pas, non plus, une création du Mercure François comme en atteste l'existence de chroniques médiévales dédiées à l'histoire de villes sur plusieurs générations et dont le succès ne se dément pas à l'époque moderne (Coulomb, 2010 : 11). L'originalité réside ici plus dans la petite échelle adoptée par le Mercure François pour son histoire que dans la périodicité qui rythme sa parution. Le point de départ du récit est la Chronologie septenaire de Cayet, et lorsque Jean Richer disparait à la fin des années 1620, son frère et collaborateur Estienne poursuit seul cette entreprise avant que d'autres rédacteurs et imprimeurs ne poursuivent la publication (Olivier de Varennes, Pierre Billaine, Jean Hénault) livraison après livraison.

Plus qu'une publication périodique, le Mercure François est une publication continue, qui, en participant à la fondation d'une mémoire du royaume assure des fonctions politiques.

\section{L'écriture historienne du temps présent dans le Mercure François. Normes, formes et pratiques}

\section{Alathie et Apathie : les deux compagnes du Mercure François ${ }^{9}$}

13 Faire de la vérité et de la neutralité ses compagnes de route au gré de l'écriture se justifie par la fonction assignée à l'histoire (Cayet, 1605 : s.f.). Estienne Richer y revient dans l'avertissement au lecteur du deuxième volume (MF vol. II, 1613 : s.f.). Chacun doit pouvoir accéder à l'histoire grâce à la simplicité du propos, qui rapporte les faits tels qu'ils eurent lieu et permet à chacun d'exercer son esprit critique. L'histoire est une invitation au positionnement juste. Ce dernier découle de l'exposition des faits. Si l'écriture du passé respecte ces principes, sa lecture sera didactique voire prescriptive. La 
vérité inhérente à l'écriture historienne est vertueuse en cela qu'elle rend le lecteur lucide et encourage à l'adoption de comportements vertueux. Le Mercure assume une fonction édificatrice qui évoque celles des trois genres rhétoriques (le délibératif, le judiciaire et l'épidictique) définis par Aristote en se départissant des ornements oratoires propres à ces genres. L'écriture du passé joue donc un rôle politique :

Ainsi sous ce nom de Continuation du Mercure (...), l'Autheur de ce Recueil espere que l'on s'en servira comme d'une guide \& adresse à tenir le chemin certain. (...) [parce qu'] en lisant les Histoires chacun peut sans longue estude voir les actions vertueuses des grands \& des petits : ce qui incite tellement les esprits à la vertu, que ceux qui les lisent bien, detestent le vice \& la rebellion, (...) tiennent le certain chemin de la vertu pour vivre en gens de bien (...) (MF vol. II, 1613 : s.f.)

Il est arrivé que le Mercure contrevienne au principe de vérité. C'est en tout cas l'avis du pouvoir royal lorsque, le 7 août 1612, il censure l'ouvrage et interdit la poursuite de la publication en raison de son caractère dangereux. C'est le récent et fragile régime de paix du royaume qui est menacé d'après le parquet. Dans l'arrêt de la cour de Parlement qui publie la décision de la justice royale en forme de factum judiciaire, l'ouvrage de Richer est qualifié par le pouvoir de mauvaise histoire. Pour les gens du roi, certaines des nouvelles publiées dans le Mercure François donnent à penser que :

(...) il y a disette d'hommes sçavants, \& qui puissent mettre la main à la plume pour composer une bonne histoire: En quoy les doctes mains sont blessées, \& les hommes amusez par des Muses sauvages, \& plusieurs faicts publiez contre verité de ce qui a esté recueilly de fauls bruicts \& mauvais comptes (...) (Arrest de la Cour de Parlement donné en la Chambre de Edict (...), BnF, manuscrits, Richelieu, fonds français, 22087 35.)

La décision du Parlement de Paris s'explique en partie par la nécessité de neutraliser la menace d'une crise diplomatique entre le royaume de France et certains de ses alliés, parmi lesquels la ville impériale de Cologne, qui s'est offusquée de la publication de certains propos concernant la diffusion de la nouvelle de la mort d'Henri IV. Le Sénat de la Ville publie en 1611 une Apologie (...) contre les calumnies d'un certain Autheur françois (...) (BnF, manuscrits, Richelieu, fonds français, Cinq-Cents Colbert). Pour le pouvoir judiciaire, c'est la réaction des autorités colonaises qui prévaut sur le respect des normes du genre historique, comme le prouve l'interdiction du Mercure. Le destin du Mercure François ne s'est pas arrêté à cet épisode judiciaire de l'été 1612. Jean Richer négocie avec le pouvoir royal la poursuite de son travail en promettant de corriger le texte du Mercure dans ses éditions prochaines. Le marché est accepté et les éditions suivantes épurées (MF vol. I, 1619 : s.f.). Si les positions du pouvoir et celles du rédacteur ont pu entrer en contradiction en 1612, leurs conceptions théoriques de l'écriture du passé se rejoignent autour de l'exigence de vérité. Ce principe est maintenu par les rédacteurs du Mercure les sept fois qu'un volume est préfacé. L'intérêt de la publication du passé réside dans l'idée que l'histoire est vérité et que cette vérité prescrit nécessairement les justes maximes politiques aux sujets du royaume; et donc les bons comportements. Dans son adresse au lecteur, Estienne Richer compare les « Maximes d'Estat » présentes dans les Chroniques de Joinville à celles que le lecteur trouvera dans son propre ouvrage (MF vol. II, 1613 : s.f.). C'est à l'édification politique du lecteur que sert l'archivage du présent et l'écriture du passé. En cela encore, le Mercure François hérite de pratiques traditionnelles.

L'Apathie, ou absence de passion, doit aussi caractériser l'activité de l'archiviste du temps. Elle est le corollaire de la vérité. Comme l'exhaustivité, elle en découle. L'historien ne peut cacher une partie des faits dans le but d'orienter ses lecteurs (MF vol. VIII, 1623 : 
s.f.). Cette position est pour le moins ambiguë surtout lorsque l'on sait que Jean Richer a censuré les passages jugés délictueux par le pouvoir. Le rédacteur se justifie parfois de s'être montré exhaustif afin de servir la vérité des faits sans endosser personnellement de prise de position, et peut-être aussi pour se prémunir des reproches de la justice :

Si j'ay rapporté en quelque endroict quelque traict, ç’a esté plutost suivant qu'un chacun avoit en la bouche au temps que les choses descrites ce sont passees, que d'aucune passion que j'eusse de ma part. Mon dessein a toujours esté de n'offenser personne(...) (MF vol. IV, 1617 : s.f.)

17 En dépit de ses déclarations d'intention, le Mercure n'est pas toujours neutre. Les vertus édificatrices de la vérité rendent parfois inutiles les faux-semblants et c'est en toute sincérité que les rédacteurs livrent leur sentiment sur une affaire dont ils pensent qu'elle ne peut faire que consensus ${ }^{10}$. C'est, en quelque sorte, parce que la vérité n'est pas la neutralité et qu'elle est située politiquement du côté de l'obéissance au pouvoir monarchique. Cette prétendue neutralité prend la forme d'un engagement politique au moment des guerres de religion; celui du Tiers-Parti fidèle au pouvoir royal ${ }^{11}$. À cette occasion, les principes traditionnels de l'écriture du passé rencontrent les convictions du parti des politiques et les intérêts du pouvoir royal. Rencontre qui nécessite parfois des ajustements pour finir par être volontairement prise en charge par le pouvoir royal de façon certaine à partir de 1639 (peut-être dès 1637) lorsque Théophraste Renaudot prend la direction du périodique ${ }^{12}$.

Les normes éthiques qui président ouvertement à l'écriture du Mercure François sont héritées de pratiques traditionnelles de l'écriture historiographique. Elles revêtent des fonctions politiques et s'adaptent sans cesse au contexte.

\section{Mise en ordre du temps et composition du recueil}

19 Les choix en matière de composition du recueil ont pour conséquence d'ordonner le temps qui n'a de cesse de s'écouler. Les titres successifs des différentes livraisons de la collection montrent la perpétuelle adaptation des compilateurs au contexte politique du royaume. La première édition du premier volume préfère s'affranchir d'une organisation par règne et repousser la borne ante quem de l'ouvrage au sacre de Louis XIII, marquant ainsi un continuum dynastique et politique entre le règne d'Henri IV et celui-de son fils malgré le régicide du mois de mai 1610, ou plutôt en raison du régicide. L'histoire qui est alors proposée n'est ni une histoire du règne d'Henri IV, ni une histoire du règne de Louis XIII, ni une histoire de la régence de Marie de Médicis mais une Histoire de la paix. En cela, elle est bien la consignation d'un temps passé depuis 1598 mais aussi du présent, et une exhortation à un futur apaisé. Au moment où le premier volume du Mercure est édité, le régime de paix se poursuit dans le royaume. Le Mercure François semble avoir échappé ici à la tyrannie de la chronologie qui s'exerce sur les chroniques et les annales mais aussi à la tyrannie du règne (Guenée, 1973 : 1007). C'est vrai parce que l'enjeu politique de la mise en exergue d'un continuum politique entre les deux règnes dépasse, en 1611, la nécessité ou l'habitude d'ordonner les temps en fonction des règnes. En 1613, le Mercure François devient la suite de l'Histoire de l'Auguste Régence de la Royne Marie de Médicis. Dans le troisième volume publié en 1616, le Mercure François est divisé en deux, parce que le temps politique l'est également (MF vol. III, 1616: 605). Louis XIII devient politiquement majeur dans le temps du troisième volume, soit au cours de l'année 1614. C'est pourquoi le traitement de l'année 1614 se structure matériellement en fonction de ces deux périodes politiques. Une page de titre est intercalée entre ces deux moments politiques de l'année. 
Elle précise : «Commencement de la Majorité » (MF vol. III, $1616: 1$ ) et la pagination reprend à zéro. Entre le second tome de cette troisième livraison et la pénultième en 1647 , le Mercure François est le récit continu de la Suite de l'Histoire de Nostre temps sous le Règne du Très-Chrestien Roy de France \& de Navarre Loys XIII. L'archivage du présent et l'écriture du passé s'actualisent par les mêmes pratiques et s'inscrivent dans une double temporalité. D'une part, dans un temps politique clôt à la fin de chaque règne ${ }^{13}$. Les circonstances politiques ne justifient plus de contrevenir aux normes d'écriture de l'histoire du temps présent, c'est-à-dire à la convention selon laquelle une telle histoire s'organise en fonction des règnes ${ }^{14}$. D'autre part, dans le temps individuel puis collectif des contemporains des règnes successifs, comme si le temps du pouvoir politique se fondait dans le temps du politique et donc de la vie de la cité. De ce point de vue, le Mercure François renvoie à la complexité du genre de la chronique ainsi qu'au statut de témoin de l'historien qui sait les choses « pour les avoir vuës » (Cayet, 1605 : s.f.).

Ainsi, dans l'organisation de la collection et des recueils, la mise en ordre du temps s'émancipe d'une chronologie qui serait dictée par les seules années pour s'inscrire dans un continuum temporel englobé par la collection. Certes, les volumes restent essentiellement organisés par année et des pages de titres ou des sommaires sont insérés dans les volumes lorsque l'on passe de la relation d'une année à une autre ${ }^{15}$. Cependant, si pour les premières livraisons l'année est systématiquement précisée en haut de chaque feuillet, rapidement (à partir du moment où l'on entre dans l'Histoire de notre temps, au tome trois donc) ce n'est plus le cas. Dès lors, l'on note l'absence de notifications pour les volumes trois et quatre alors que le numéro du tome est précisé sur quelques pages des livraisons suivantes à partir de la cinquième, aux pages 17 ou 49 par exemple. C'est le cas jusqu'au vingtième tome inclus, avant que Renaudot ne parvienne à la tête de la publication, probablement en 1637. La foliotation est partiellement abandonnée au profit d'une pagination qui ne se fait pas en fonction des années mais des tomes ${ }^{16}$. Le temps découpé en années successives perdure parce qu'il n'est pas possible de l'ignorer, mais, là-encore il est subsumé par le temps de la collection qui vient le recouvrir. Il dépend de ce temps vécu des individus entre présent et futur passé et se soumet à la collection qui ordonne le temps en son temps propre, un « notre temps $»^{17}$.

21 C'est la logique de la collection qui transforme le temps présent des contemporainstémoins en un passé et participe à rapprocher le Mercure François de la chronique.

\section{Les matériaux de la compilation, sources de l'Histoire de nostre temps}

22 La mise en ordre du temps que nous venons d'évoquer passe aussi par le travail de compilation. Cette démarche renvoie à des pratiques d'écriture du temps partagées par les différents genres historiques, y compris la chronique (Guenée, 2011: 212). Johann Petitjean identifie les diverses étapes qui constituent le travail de la compilation. Il s'agit d'un acte de « lire-écrire » en cela que le compilateur doit sélectionner des extraits dans un large corpus avant de les ordonner en une unité raisonnée (Petitjean, 2009a: 18). Il souligne que c'est au XIII ${ }^{\mathrm{e}}$ siècle que la compilation connaît son âge d'or (Petitjean, 2009a : 21). La compilatio est alors reconnue comme une forme d'écriture à part entière. La dimension d'intertextualité qu'elle suppose permet une mise en ordre de temporalités diverses. Celle du présent et des actualités, lorsque les étapes préparatoires sont rapides; la compilation peut alors être manuscrite et donner lieu à la diffusion de 
nouvelles à la main (Petitjean, 2009b : 81). Celle du passé également, dans le cas d'un temps de lecture et de constitution du recueil plus long, à partir de sources dont l'impression a elle-même conduit à l'écoulement d'un temps assez long. La compilation apparait comme l'une des formes naturelles de l'écriture historiographique.

Les considérations d'un Cayet ou d'un Pierre Matthieu, historiographe du roi, à propos des sources et références de l'historien le font sortir de son statut de témoin. Il accepte de faire confiance à ses prédécesseurs et de mêler ses propres témoignages à la compilation de textes historiques. C'est la nécessité qui l'explique et qui conduit souvent les historiens du XII e siècle à intituler leurs ouvrages "fleurs des histoires ", "fleurs des chroniques " ou encore "fleurs des temps" (Guenée, 2011: 211-212). Estienne Richer affirme que l'auteur de la seconde livraison du Mercure a «(...) recueilly les fleurs des plus belles relations de ce qui s'est passé depuis la Regence de la Royne » (MF vol. II, 1613 : s.f.). Mais, quelles sont-elles, ces fleurs?

Le Mercure François se nourrit d'une grande intertextualité mais cite peu de sources précises. La masse constituée par les 25 volumes de la collection permet toutefois d'en glaner quelques-unes. Le tout premier volume, particulièrement le traitement de la mort d'Henri IV, renvoie à d'autres travaux d'historiens. Le travail de Cayet e st cité, comme celui de Pierre Matthieu. L'intertextualité est en partie historique. Dans le même temps, le rédacteur n'hésite pas à faire part de ses propres témoignages et à se placer en témoin oculaire (MF vol. I, 1611 : f ${ }^{\circ} 430 \mathrm{r}^{\circ}$.). Les autres sources citées ou reproduites sont ensuite des correspondances, des sources officielles comme les arrêts de la cour interdisant les duels (MF vol. X, 1625 : 391) ou encore des pièces de guerres de plume comme c'est le cas à propos d'un désaccord protestant au moment de la reprise des guerres civiles (MF vol. VIII, 1623: 154). Néanmoins, les références précises fournies par les rédacteurs évoluent au fil des livraisons. Les volumes sept à dix mentionnent à plusieurs reprises le Mercurius Gallobelgicus qui semble être la source d'informations des rédacteurs pour l'est et le nord de la chrétienté ${ }^{18}$ (MF vol. VII, $1622: 7 ; 30 ; 178 ; 759$; vol. VIII, $1623: 234$; vol. IX ,1624: 59). Une des sources de la compilation des frères Richer est ici une autre compilation qu'il a fallu traduire ${ }^{19}$. Au travail habituel de la compilation vient s'ajouter un travail de traduction pour apporter une connaissance de contrées éloignées aux lecteurs du royaume. L'intertextualité du Mercure François est donc historique et ses références à un ouvrage similaire se doublent d'un système d'autoréférenciation qui fait du Mercure sa propre source C'est ainsi que le rédacteur renvoie ses lecteurs à d'autres volumes du Mercure en indiquant les pages ou les folios, en marge ou au fil du texte, à la faveur d'une transition par exemple. On peut le lire à propos du duel Montmorency-Bouteville :

Nous avons dit au Tome 10 du Mercure François p. 385 que ny les Edicts sur les duels ny les Justices exemplaires executees contre les contrevenans n'avoient peu arrester ceste ardeur de sang qui porte plusieurs de la Noblesse Françoise à se rendre sur le pré, pour y tirer raison de leurs querelles. (MF vol. XIII, 1628: $400 \mathrm{ou}$ MF vol. XIX, 1636: 8).

Comme pour la formation d'un temps propre au périodique, ce double système de référenciation consacre la logique de la collection et en fait un système d'information et de mise en ordre du temps. Néanmoins, lorsque l'on fait un saut dans le temps et que l'on passe du volume dix au volume quatorze les références explicites au Mercurius Gallobelgicus disparaissent alors que l'ouvrage est publié jusqu'en 1638. Dans le même temps, le système d'autoréférenciation se fait plus discret. Les autres références précises mentionnent désormais des témoins directs et des relations venues de correspondants. Les références historiques de relations du passé laissent la place à des textes toujours 
décalés dans le temps, mais qui fondent plus une écriture de l'actualité que du passé. On retrouve ici le statut de témoin de l'historien. Apparaissent ainsi dans les pages du Mercure des expressions telles que « Les Relations d'Allemagne nous apprennent (...) » (MF vol. XVI, 1632:906) ou "Voyons ce que nous avons peu recouvrer, cette annee, des Relations de Constantinople» (MF vol. XVI, 1632:1032). Les auxiliaires à l'œuvre de compilation ne sont plus des témoins oculaires d'un passé assez lointain, mais ceux d'une actualité distante géographiquement du royaume. L'histoire redevient une histoire du temps présent et l'historien, son témoin. La tendance s'accentue sous la direction de Renaudot qui applique au Mercure les méthodes d'investigation éprouvées dans la Gazette et sollicite ses correspondants locaux, comme on le lit ici, lorsqu'il introduit des nouvelles de Rome : "Nous vous faisons donc part de leur récit particulier, ainsi qu'il nous a esté envoyé » (MF vol. XXII, $\left.1641: 318^{20}\right)$. La mise en ordre de l'espace vient accompagner voire recouvrir celle du temps et si le recueil ne prend jamais tout à fait la forme de la Gazette qui classe ses informations selon leurs lieux de production, la logique spatiale devient indéniable dans les dernières livraisons (MF vol. XXII, 1647 : 377). Le temps du récit est de plus en plus proche de celui de la collecte des informations. Pour autant, la publication des dernières livraisons du Mercure est retardée par rapport aux événements relatés, la périodicité perd en régularité avant de disparaître. La primeur est réservée à la Gazette et, tout en prenant certaines des formes de la presse hebdomadaire (selon le modèle de fournie par la Gazette), le Mercure François est en fait condamné à disparaître. En effet, le voilà cantonner à devenir un genre historique qui aurait perdu ses spécificités tout en échouant à participer pleinement au genre de la presse ; en raison de la péremption des nouvelles qu'il publie à retardement et après la Gazette. À cet égard, le Mercure François pourrait être considéré comme l'un des nombreux genres « caducs » à avoir traversé son temps au prix de transformations (Montandon et Neiva, $2014: 15$ ).

Les références fournies par les rédacteurs successifs du Mercure témoignent d'une évolution dans la pratique de la compilation et d'une plasticité du périodique qui conserve pour activité principale la mise en ordre du ou des temps.

\section{Conclusion}

Le Mercure François, parce qu'il prend pour objet le temps réunit, des pratiques d'écriture qui s'apparentent à celles de la chronique. Il est difficile d'imposer au Mercure François une catégorie générique stricte et définitive. Le Mercure François est une histoire du temps présent. À ce titre, il est à la fois écriture du passé et écriture du présent. Son objet comme ses usages se situent au carrefour des temporalités. Ses pratiques d'écriture relèvent d'une longue tradition de saisie et de mise en ordre d'un temps politique - ou d'un temps du politique - à laquelle la chronique a, aussi, contribué. Les pratiques d'écriture héritées d'une tradition de mise en récit du temps sont nombreuses à être partagées avec celle de la chronique. C'est le cas du choix de la langue vernaculaire ou de la conception continue du temps. C'est l'usage politique de cette mise en ordre du temps qui dicte des évolutions à la chronique comme au Mercure François. Changements de rythme ou changement des sources convoquées pour la compilation s'expliquent par les usages politiques investis sur le temps. C'est également à ce titre que les circonstances politiques ont des effets sur le Mercure François et compliquent encore l'exercice du classement par genres. Par conséquent, la plasticité générique et disciplinaire des écritures du temps doit conduire à s'interroger sur les évolutions de leurs usages. La 
récupération certaine du Mercure François par le pouvoir politique en la personne de Renaudot à la fin des années 1630 en est une belle illustration. Le pouvoir s'est infiltré au sein de cet organe de mise en ordre du temps politique et peut le surveiller de plus près si ce n'est le diriger. Les pratiques d'écriture s'infléchissent alors un peu plus. Le Mercure François devient rapidement caduc puis disparait, sans doute en raison des altérations subies et des usages commerciaux de l'écriture du temps par Renaudot. Pourtant, comme d'autres genres caducs (Montandon et Neiva, 2014:10-11), le genre éteint en 1648 semble partiellement réinvesti comme en atteste par exemple l'apparition d'un nouveau type de publications en Europe dans la seconde partie du XvII ${ }^{\mathrm{e}}$ siècle. Cette fois-ci, la référence à Mercure s'ancre plus fermement dans l'actualité et désigne des mensuels qui fleurissent avec succès à partir du dernier tiers du XVII ${ }^{e}$ siècle. L'histoire n'est cependant pas absente de ce nouveau genre, celui des «Mercures historiques et politiques» (Brétéché, 2013 : 49-63).

\section{BIBLIOGRAPHIE}

\section{Sources manuscrites}

Apologie du Senat de la Ville imperiale et libre de Colongne contre les calumnies d'un certain Autheur François sans nom lequel entre autres choses par luy faulsement mises en avant contre ledict Senat, faict mention en ses Annales, que le meurtre du Roy tres-Chrestien Henry quastriesme Roy de France auroit esté loüé publiquement, \& en pleine predication, ce consentant \& approuvant le Magistrat de ladite ville. A Colongne, Par Jean de Mertzenich, Anno M. DCXI, BnF, manuscrits, Richelieu, Cinq-Cents Colbert, vol. $12, f^{\circ} 104 v^{\circ}-110 v^{\circ}$.

Arrest de la Cour du Parlement donné en la Chambre de l'Edict entre Jean Richer Libraire \& Autheur du Libvre intitulé Le Mercure François, ou suite de l'histoire de la Paix demandeur en requestes \& defendeur d'aultre part, Et Adrian Perier aussi Libraire defendeur \& aussi demandeur en requeste d'aultre par lequel la Cour a ordonné que ledict Libvre sera supprimé, BnF, manuscrits, Richelieu, fonds français, 2208735.

\section{Sources imprimées}

MATTHIEU Pierre (1613), Histoire de France \& Des choses memorables advenues aux Provinces estrangeres durant sept annes de Paix DU REGNE De Henry IIII Roy de France \& de Navarre. DIVISEE EN SEPT LIVRES (M. DC. XIII). A PARIS chez J. Metayer imprimeur du Roy \& M. Guillemot au Palais en la Gallerie des prisonniers.

PALMA-CAYET Pierre-Victor (1605), Chronologie septenaire de l'Histoire de la paix entre les Roys de France et d'Espagne. Contenant les choses plus memorables advenuës en France, Espagne, Allemagne, Italie, Angleterre, Escosse, Flandres, Hongrie, Pologne, Suece, Transsilvanie, \& autres endroits de l'Europe : avec le succez de plusieurs navigations faictes aux Indes Orientales, Occidentales \& Septentrionales, depuis le commencement de l'an 1598. Jusques à la fin de l'an 1604. DIVISEE EN SEPT LIVRES. Troisiesme Edition, A 
Paris, Par Jean Richer, ruë S. Jean de Latran à l'Arbre Verdoyant : Et en sa boutique au Palais, sur le Perron Royal, vis à vis de la gallerie des Prisonniers, M.D.CVII.

MERCURE FRANÇOIS (MF) (1611 à 1648), volumes I à XXV.

\section{Bibliographie}

BRÉTÉCHÉ Marion (2013). « Entre actualité et histoire : le pari des Mercures historiques et politiques (1686-1730) », in LÉVRIER Alexis et WRONA Adeline (dir.), Matière et esprit du journal. Du Mercure Galant à Twitter. Paris : PUPS.

BRÉTÉCHÉ Marion (2012). Les compagnons de Mercure. Écrire et publier l'information politique européenne. Provinces-Unies - France (1680-1740). Thèse inédite de Doctorat en Histoire moderne soutenue le 24 novembre 2012 sous la direction de Monsieur Lucien BÉLY.

coulomb Clarisse (2010). «Des villes de papier. Écrire l'histoire de la ville dans l’Europe moderne ", Histoire urbaine, $n^{\circ} 28$, pp. 5-16.

FEYEL Gilles (2000). L'annonce et la nouvelle : la presse d'information en France sous l'Ancien Régime, 1630-1788. Oxford : Voltaire Foundation.

GuENÉE Bernard (1973). « Histoire, annales, chroniques. Essai sur les genres historiques au MoyenÂge ", Annales, Économies Sociétés, Civilisations, $28^{\text {ième }}$ année, vol. 4, pp. 997-1016.

GUENÉE Bernard (2011). Histoire et culture historique dans l'Occident médiéval. Paris : Flammarion. HAFFEMAYER Stéphane (2002). L'information dans la France du XVII siècle : la gazette de Renaudot de 1647 à 1663. Paris : Champion.

HAFFEMAYER Stéphane (1999). La géographie de l'information dans la Gazette de Renaudot de 1647 à 1663, in RÉTAT Pierre et DURANTON Henri (éds.), Gazettes et information politique sous l'Ancien Régime, actes du colloque international de Lyon 5-7 juin 1997. Saint-Étienne : Publications de l'Université de Saint-Étienne.

JAUSS Hans-Robert (1986). « Littérature médiévale et théorie des genres », in GENETTE Gérard et al., Théorie des genres, pp. 37-76. Paris : Points Seuil.

JAUSS Hans-Robert (1988). Pour une herméneutique littéraire. Paris : Gallimard.

JoUHAUd christian (2000). Les pouvoirs de la littérature. Histoire d'un paradoxe. Paris : Gallimard.

JOUHAUD Christian, RIBARD Dinah, SCHAPIRA Nicolas (2009). Histoire Littérature Témoignage. Écrire les malheurs du temps. Paris : Folio Histoire.

JUBERT Gérard (éd.) (2005). Théophraste Renaudot (1586-1653). Père des journalistes et médecin des pauvres. Paris : Champion.

MAINGUENEAU Dominique (2007). «Genres de discours et modes de généricité », Le français aujourd'hui, n 159, pp. 29-35. Paris : Armand Colin.

MONTANDON Alain, NEIVA Saulo (dir.) (2014). Dictionnaire raisonné de la caducité des genres littéraires. Genève : Droz.

PETITJEAN Johann (2009a). « Compiler. Formes, usages et pratiques », Hypothèses, vol. 1, pp. 15-25. Petitjean Johann (2009b). « Compilation des nouvelles et écriture de l'actualité à Venise au XVI siècle ", Hypothèses, vol. 1, pp. 73 à 82.

SCHAEFFER Jean-Marie (1989). Qu'est-ce qu'un genre littéraire ?, Paris : Seuil. 
YARDENI Myriam (1971). La conscience nationale en France pendant les guerres de religion (1559-1598).

Paris-Louvain : Nauwelaerts.

YARDENI Myriam (1981). «Ésotérisme, religion et histoire dans l'œuvre de Palma Cayet », Revue de l'histoire des religions, tome. 198, pp. 285-308.

\section{NOTES}

1. Les deux formes se confondent et restent indifférenciées pendant longtemps d'après Bernard Guenée (Guenée, 1973 : 998).

2. Voir à ce propos la mise au point de Saulo Neiva (Montandon et Neiva, 2014 : 13).

3. Hans-Robert Jauss rappelle à ce propos qu'il convient de prendre des précautions méthodologiques et de ne pas tomber dans l'écueil inverse qui consisterait à ignorer totalement l'autonomie du genre (Jauss, $1986: 52-54$ ).

4. Jean Richer est l'imprimeur de Pierre-Victor Palma Cayet.

5. Jusqu'à la première moitié du XVI ${ }^{\mathrm{e}}$ siècle d'après Bernard Guenée (Guenée, 1973 : 1004).

6. C'est aussi la conception de l'historiographe Pierre Matthieu dans l'épître au roi de son Histoire de France (...) qui définit son ouvrage comme nécessaire à la fabrique d'une mémoire du règne adressée à la postérité. Bernard Guenée ajoute d'ailleurs que dans la conception médiévale « Il n'y a pas de différence de nature entre histoire et prophétie. » (Guenée, $2011: 22$ ).

7. Le Mercurius Gallobelgicus est le premier périodique européen publié à Cologne à partir de 1588. Il est écrit en latin et paraît de manière bisannuelle jusqu'en 1638. Il s'agit aussi du premier périodique à renvoyer au dieu Mercure comme le rappelle Marion Brétéché (Brétéché, 2012).

8. On peut aussi penser que le succès commercial des ouvrages de Cayet comme du premier Mercure a encouragé les libraires dans leur commerce.

9. Nous renvoyons ici au titre de la thèse de doctorat de Marion Brétéché à propos de l'écriture et de la publication politique en France et aux Provinces-Unies entre les années 1680 et 1740 . B RÉTÉCHÉ Marion, Les compagnons de Mercure. Écrire et publier l'information politique européenne. Provinces-Unies-France (1680-1740). Thèse de doctorat en histoire moderne soutenue le 24 novembre 2012 sous la direction de Monsieur Lucien BÉLY.

10. Celle de l'assassinat d'Henri IV par exemple.

11. Le Tiers-Parti aussi appelé parti Politique soutient le pouvoir royal contre les deux factions qui le remettent en cause : celle des protestants et celle des catholiques zélés.

12. Théophraste Renaudot est l'un des proches du cardinal de Richelieu. Il lui arrive de publier dans la Gazette qu'il fonde en 1631 des textes de la main du cardinal ou du roi (Feyel, 2000 : 172-177).

13. La dernière livraison du Mercure publiée en 1648 s'intitule Tome premier de l'Histoire de Nostre Temps, sous le règne du Très-Chrestien Roy de France \& de Navarre Loys XIV es 1643 \& 1644 ou Tome VingtCinquiesme du Mercure François, es mesmes années 1643 \& 1644.

14. En cela, le Mercure François applique certaines normes héritées de la chronique médiévale (Guenée, 1973 : 1007).

15. C'est le cas dans le tome 5 par exemple après la page 337.

16. L'avertissement au lecteur du tome 16 en atteste: «Bien que plusieurs memoires que nous avons en main, deussent estre inserez au Tome quinziesme de nostre Mercure : neantmoins afin de ne pas ennuyer le lecteur par la lecture d'un trop gros volume nous avons trouvé à propos de continuer par le commencement d'un Seiziesme tome. (MF vol. XVI, 1632 : s.f.)

17. Il est possible de penser ici que le nous de «notre temps » renvoie au temps vécu des lecteurs du Mercure. 
18. On retrouve dans ces numéros des renvois à Gothardus l'auteur du Mercurius Gallobelgicus ou à Londorpius qui a participé à la rédaction d'une histoire des territoires allemands également en latin.

19. Voir à ce propos la " Preface au lecteur » : « Je te donne dans ce livre toutes les choses les plus remarquables (...) que j'ay faictes Françoises à ma mode (...)» (MF vol. I, 1611 : s.f.).

20. La Gazette dont le premier numéro date de 1631 est le premier hebdomadaire français. Théophraste Renaudot en est l'initiateur.

\section{RÉSUMÉS}

En 1611 débute la publication d'une collection de périodiques intitulée le Mercure François. En raison de sa périodicité (annuelle entre 1619 et 1633), l'ouvrage est souvent considéré comme inaugurant la pratique du journalisme en France. En réalité, le Mercure François s'apparente plutôt à un ouvrage d'histoire. Il hérite d'une tradition de mise en récit du passé en prenant la suite de la Chronologie septenaire de l'Histoire de la paix [...] de Pierre-Victor Palma-Cayet. Si la distance temporelle et géographique entre les événements relatés et la publication du texte se réduit pendant un temps, le Mercure François reste un livre d'histoire. L'articulation des temporalités qu'il réalise le place au croisement de la tradition et de l'innovation et aux frontières de différents genres littéraires comme discursifs. Les bornes temporelles de publication du Mercure François entre l'assassinat d'Henri IV (1611) et la régence d'Anne d'Autriche (1648) font traverser au périodique trois régimes politiques distincts qui posent la question de la fonction politique de l'écriture du passé au regard du contexte politique.

In 1611 started the publication of a collected works of periodicals called the Mercure François. Because of its periodicity (it was published once a year between 1619 and 1633), the periodical is often regarded as the beginning of journalism in France. As a matter of fact, the Mercure François is closer to a history book. It inherits from an extended tradition of writing the past and it follows the Chronologie septenaire de l'Histoire de la paix [...] by Pierre-Victor Palma-Cayet. If the chronological and geographical distance is getting shorter and shorter during a period of time, the Mercure François is still a history book. Because of the various temporalities that it deals with, the Mercure François stands at the crossroad of tradition and innovation and could be associated to various literary and discursive genres. Its publication between the murder of Henry IV (1611) and the regency of Anne d'Autriche (1648) makes the periodical cross three political regimes thus questioning the political use of writing the past.

\section{INDEX}

Mots-clés : tradition, histoire, temporalités, usages politiques, actualités

Keywords : tradition, history, temporalities, political use, news 


\section{AUTEUR}

\section{VIRGINIE CERDEIRA}

UMR 7303 CNRS TELEMMe - Aix-Marseille Université

virginiecerdeira[at]voila.fr 